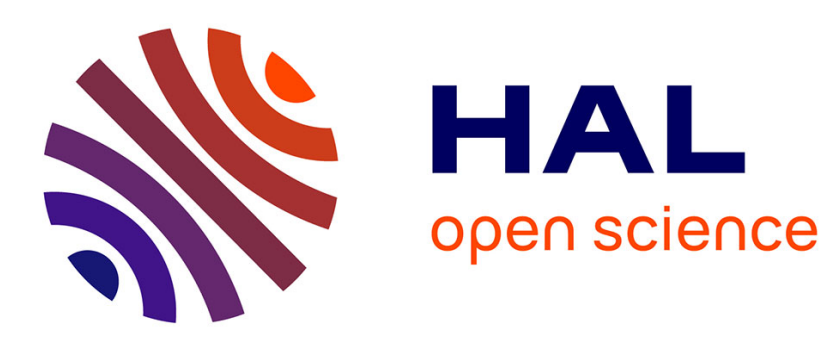

\title{
Strain and stress at the fracture of percolative media
}

\author{
Ludovic Benguigui, P. Ron, D.J. Bergman
}

\section{To cite this version:}

Ludovic Benguigui, P. Ron, D.J. Bergman. Strain and stress at the fracture of percolative media. Journal de Physique, 1987, 48 (9), pp.1547-1551. 10.1051/jphys:019870048090154700 . jpa-00210587

\section{HAL Id: jpa-00210587 https://hal.science/jpa-00210587}

Submitted on 1 Jan 1987

HAL is a multi-disciplinary open access archive for the deposit and dissemination of scientific research documents, whether they are published or not. The documents may come from teaching and research institutions in France or abroad, or from public or private research centers.
L'archive ouverte pluridisciplinaire HAL, est destinée au dépôt et à la diffusion de documents scientifiques de niveau recherche, publiés ou non, émanant des établissements d'enseignement et de recherche français ou étrangers, des laboratoires publics ou privés. 


\title{
Strain and stress at the fracture of percolative media
}

\author{
L. Benguigui $\left({ }^{1}\right)$, P. Ron $\left({ }^{1}\right)$ and D. J. Bergman $\left({ }^{2}\right)$ \\ ( ${ }^{1}$ ) Department of Physics and Solid State Institute, Technion-Israel Institute of Technology, Haifa 32000, \\ Israel \\ ( ${ }^{2}$ ) School of Physics and Astronomy, The Raymond and Beverly Sackler Faculty of Exact Sciences, Tel Aviv \\ University, Tel-Aviv 69978, Israel
}

(Reçu le 18 février 1987, accepté le 26 mai 1987)

\begin{abstract}
Résumé. - La déformation d'une feuille de métal perforée et d'un réseau élastique dilué ainsi que la contrainte ont été déterminées au voisinage du seuil de percolation, d'une part en augmentant régulièrement la déformation jusqu'à la rupture et d'autre part en augmentant régulièrement la contrainte appliquée. On a déterminé l'exposant critique $T_{\mathrm{f}}$ de la contrainte $\sigma_{\mathrm{f}}$ à la rupture $\left(\sigma_{\mathrm{f}} \sim\left(p_{\mathrm{c}}-p\right)^{T_{\mathrm{f}}}\right), T_{\mathrm{f}}=2,5 \pm 0,4$ et l'exposant critique $T_{\mathrm{u}}$ de la déformation $\varepsilon_{\mathrm{f}}$ à la rupture $\left(\varepsilon_{\mathrm{f}} \sim\left(p_{\mathrm{c}}-p\right)^{-T_{\mathrm{u}}}\right), T_{\mathrm{u}}=1,4 \pm 0,2$. Une interprétation théorique des résultats est proposée.

Abstract. - We measured the strain and the stress of a perforated metal foil and of a diluted elastic network
near the percolation threshold. We used two different techniques : in the first, the strain is increased
continuously and monotonically until the system ruptures, whereas in the second, it is the stress which is
increased monotonically. We determined the critical exponent of the fracture stress $\sigma_{\mathrm{f}} \sim\left(p_{\mathrm{c}}-p\right)^{T_{\mathrm{f}}}$, with
$T_{\mathrm{f}}=2.5 \pm 0.4$ and the critical exponent of the fracture strain $\varepsilon_{\mathrm{f}} \sim\left(p_{\mathrm{c}}-p\right)^{-T_{\mathrm{u}}}$ with $T_{\mathrm{u}}=1.4 \pm 0.2$. A
theoretical interpretation of the results is proposed within the framework of the nodes-links-blobs picture.
\end{abstract}

In this paper, we present the results of an experimental determination of the evolution of the stress and the strain of a percolative medium in two dimensions, as it is stretched through a sequence of plastic and fracture transitions until complete rupture occurs. Recently, interest has grown in the problem of fracture in a medium in which increasing amounts of non-linear and irreversible damage is created by mechanical stress up to a point where total rupture occurs [1-4]. While it is now well established that the linear elasticity of real perforated solids and that of diluted networks with bond-bending forces belong to the same universality class, it is not clear whether the fracture phenomenon also exhibits a similar universality: it is possible that the properties of the medium itself will be of importance. It is well known that for stresses above the linear regime, different behaviours are observed in brittle and in plastic materials.

First, we have to define the stress and the strain at the fracture point unambiguously. Two methods can be devised to rupture a two-dimensional sample; i.e. a sheet of the chosen material. In the first situation, the strain is increased and one measures the corresponding stress. Since near the percolation threshold the fracture takes place through breaking of a finite number of bonds, one measures the stress at the first bond breaking, at the second, and so on. One can define the fracture stress in different ways, such as either the stress at which the first bond breaks, or the largest value of stress. In our experiment this causes no problem, because we always found that the stress at the first break is also the largest stress. Thus we adopted this definition of the fracture stress. The fracture strain is defined as the relative elongation of the sample when it ruptures into two separate pieces. At that time, the stress drops to zero.

In the second situation, we impose the stress, which is increased until the breaking of the different bonds begins. Here too, one can imagine different possibilities. It is possible that once one bond is broken others immediately follow until complete rupture occurs. Another possibility is that after one bond is broken, one has to increase the force further in order to break another bond, and this continues 
until the force is sufficient to fracture the sample. As shown below, we always observed only the first possibility. Here too, the fracture strain can be defined as the relative elongation when the rupture occurs. We note that the definition of the fracture strain is always free of ambiguity, which may not be the case for the fracture stress if the first break does not occur at the largest value of stress.

The material used in this experiment is a copper foil of thickness $0.2 \mathrm{~mm}$. We performed two types of experiments, following the two procedures described above, and we found complete agreement between them. In the first type [4] we used sheets of size $20 \times 21 \mathrm{~cm}$ in which we randomly punched holes of $1.1 \mathrm{~cm}$ diamter on a square lattice of $1 \mathrm{~cm}$ unit cell. The sample was elongated in time by means of a small motor and we increased very slowly $(0.05 \mathrm{~mm} / \mathrm{s})$ the sample length. At the same time, we recorded the stress by means of a pressure sensor (Fig. 1). The time $t_{\mathrm{c}}$ of the fracture is thus proportional to the strain. In figures 2 and 3 we plotted the fracture stress $F_{\mathrm{c}}$ (the stress at the first bond breaking) and the fracture time $t_{\mathrm{c}}$, versus $p_{\mathrm{c}}-p$, where $p$ is the hole fraction and $p_{\mathrm{c}}$ is the

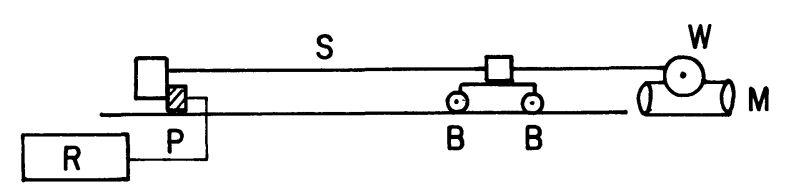

$$
\begin{aligned}
& \text { P - Pressure sensor } \\
& \text { R - Recorder } \\
& \text { S - Sample } \\
& \text { B - Ball bearing } \\
& M-\text { Motor } \\
& \text { W - Wheel }
\end{aligned}
$$

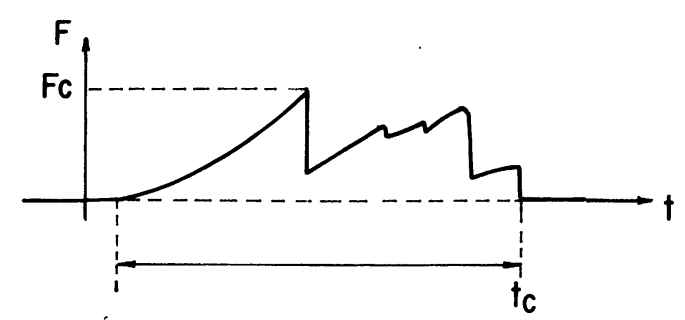

(b)

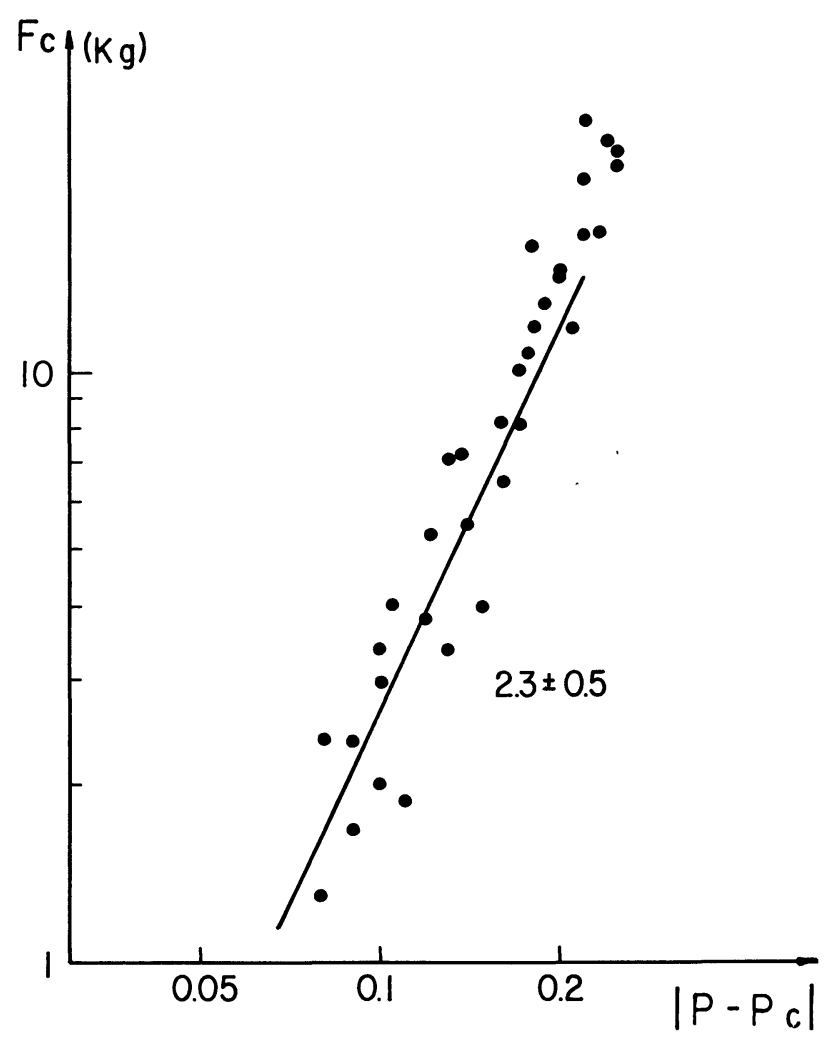

Fig. 2. - The fracture stress $F_{\mathrm{c}}$ versus $\left(p_{\mathrm{c}}-p\right)$ in a log-log plot.

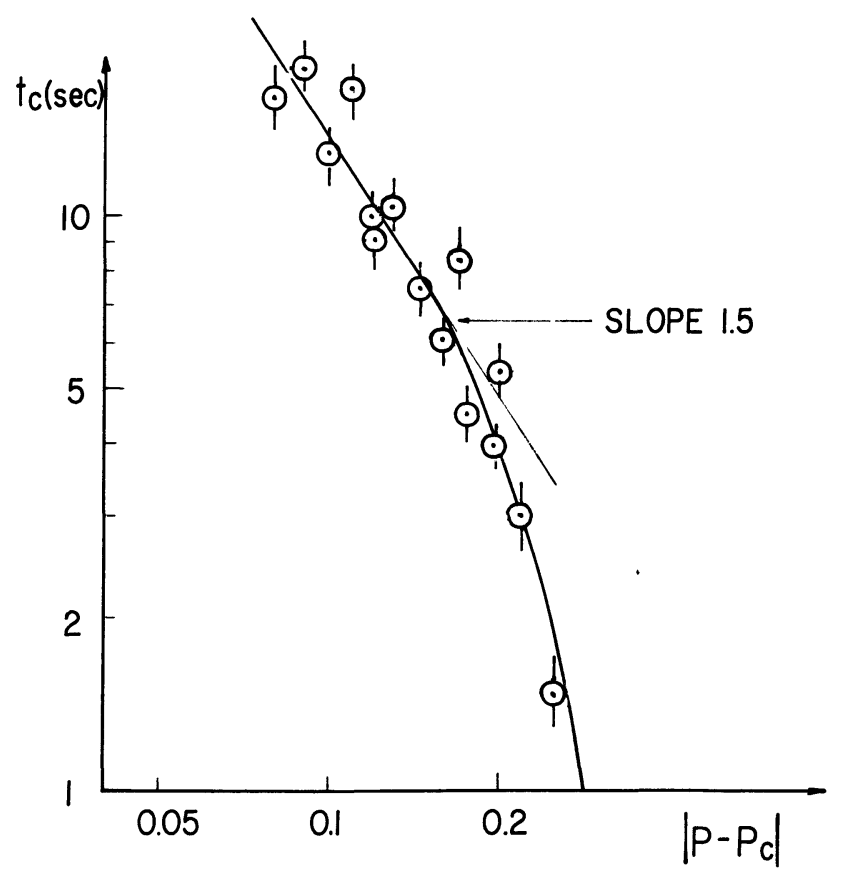

Fig. 3. - The fracture time $t_{\mathrm{c}}$ versus $\left(p_{\mathrm{c}}-p\right)$ in a log-log plot. $t_{\mathrm{c}}$ is proportional to the strain.

threshold [5] $\left(p_{\mathrm{c}} \simeq 0.6\right)$. We see that $F_{\mathrm{c}}$ goes too zero like $F_{\mathrm{c}} \sim\left(p_{\mathrm{c}}-p\right)^{T_{\mathrm{f}}}, T_{\mathrm{f}}=2.3 \pm 0.5$ and $t_{\mathrm{c}}$ diverges like $t_{\mathrm{c}} \sim\left(p_{\mathrm{c}}-p\right)^{-T_{\mathrm{u}}}, T_{\mathrm{u}}=1.5 \pm 0.5$. Each measurement was made on a different sample and in
Fig. 1. - a) Experimental set-up of the fracture experiment when the sample is elongated. b) Record of the stress versus the time during the fracture process. Each discontinuity corresponds to the breaking of a bond. $F_{\mathrm{c}}$ is the stress at the first breaking and is the largest of the stress at the different bond breakings. The initial curvature of the $F(t)$ curve is due to the fact that, at the beginning the sample is not stretched because of its loose shape. This curvature is not observed after each bond breaking. 
the case of the same value of $p$, this corresponds to a different random distribution of holes.

In the second experiment, we applied a known force (as in Ref. [6]) and measured the elongation of the sample. The difference from the set-up of reference [6] is that the elongation is determined using a different technique : in the present case a ferrite rod is monted on the side of the sample to which the force is applied. This rod can move inside the two coils of a small transformer, and an AC voltage $(1 \mathrm{khz})$ is applied to one of the coils. The magnitude of the voltage induced in the second coil, depends on the position of the rod inside the coils. This voltage is rectified and recorded through a DC amplifier (Fig. 4).

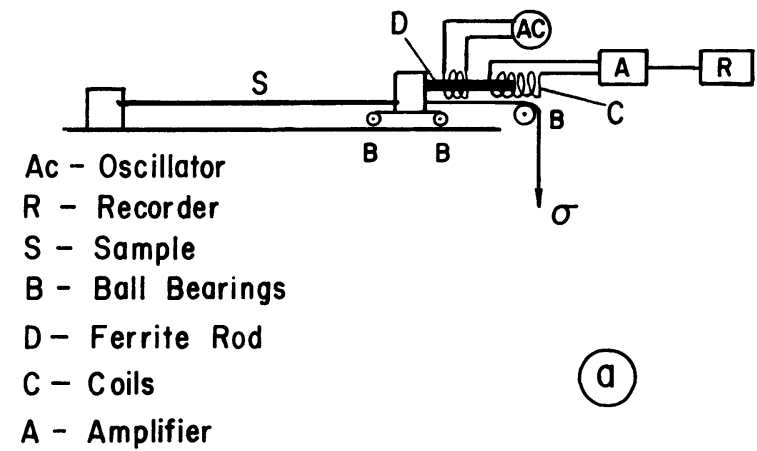

(b)

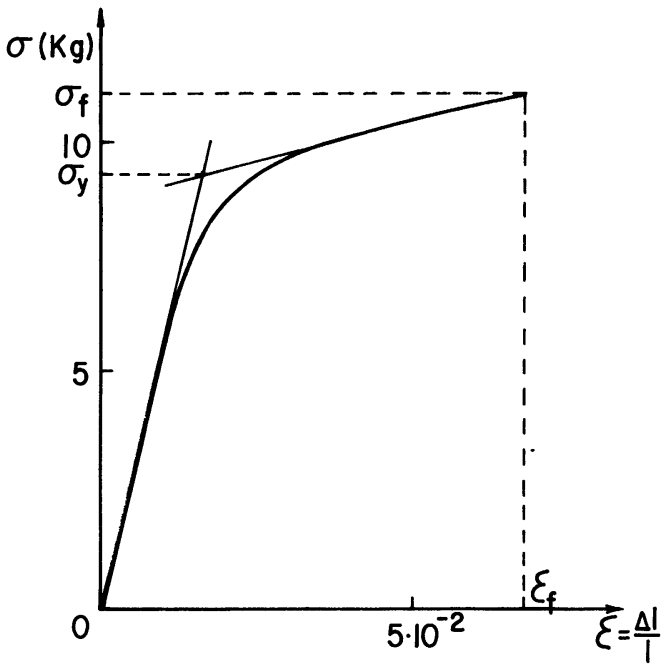

Fig. 4. - a) Experimental set-up when the stress is increased. b) The stress $(\sigma)$ - strain $(\varepsilon)$ curve until the fracture $\left(\sigma_{\mathrm{f}}, \varepsilon_{\mathrm{f}}\right) . \sigma_{\mathrm{y}}$ is the plastic yield stress.

As in the preceding experiment, the mobile side of the sample is mounted on four ball bearings in order to keep it always parallel to the clamped side. The sample is made of copper, but it is different from the sample of the first experiment : we first made a uniform square lattice of $900(30 \times 30)$ holes in the sheet. Defects were then introduced by cutting the interhole bonds at random. The measurements were made in the following way : we apply a known force and measure the corresponding strain. The force is then increased by smaller and smaller increments, until the sample ruptures into two pieces. The strainstress curve can be drawn up to the point of fracture (Fig. 4). Of course, the fracture takes place through successive breaking of several bonds. This feature cannot quantitatively be observed in the present experiment because the fracture of the sample occurs rapidly.

In figures 5 and 6 , we show the two basic quantities : the fracture stress $\sigma_{\mathrm{f}}$ and the fracture strain

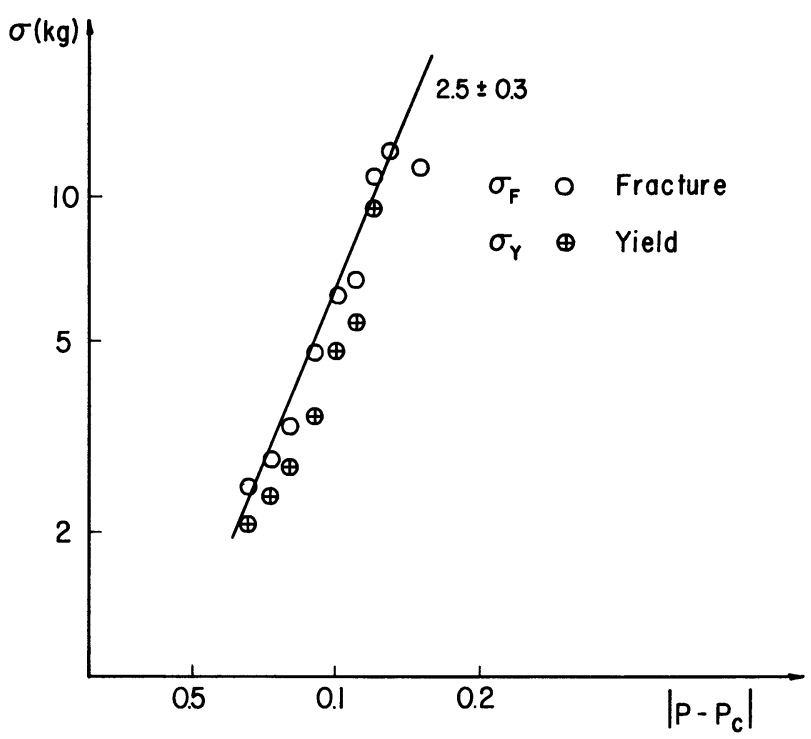

Fig. 5. - The fracture stress $\left(\sigma_{\mathrm{f}}\right)$ and the yield stress $\left(\sigma_{\mathrm{y}}\right)$ versus $\left(p_{\mathrm{c}}-p\right)$ in a $\log -\log$ plot.

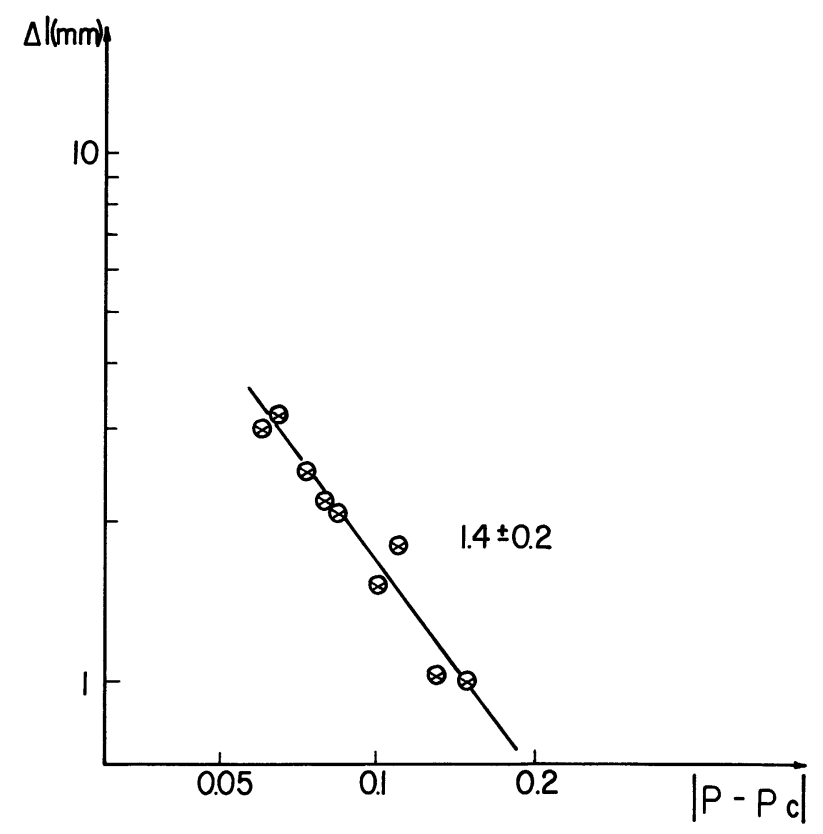

Fig. 6. - The sample elongation versus $\left(p_{\mathrm{c}}-p\right)$ in a log$\log$ plot. 
$\varepsilon_{\mathrm{f}}$ versus $\left(p_{\mathrm{c}}-p\right)$, where $p$ is the fraction of disconnected bonds and $p_{\mathrm{c}}=0.50$. As above, we determined the critical indices $T_{\mathrm{f}}=2.5 \pm 0.4$ and $T_{\mathrm{u}}=1.4 \pm 0.2$, and they are in very good agreement with the values determined in the first experiment. In figure 5, we show also the yield stress (defined in Fig. 4), versus $\left(p_{\mathrm{c}}-p\right)$ and one sees that $\sigma_{\mathrm{y}}$ behaves in the same manner as $\sigma_{\mathrm{f}}$.

Ray and Chakrabarti [1] have recently proposed a lower bound for $T_{\mathrm{f}}$

$$
T_{\mathrm{f}} \geqslant\left(T+\left(d-d_{\mathrm{B}}\right) \nu\right) / 2
$$

where $T$ is the elasticity exponent, $d$ the dimensionality, $d_{\mathrm{B}}$ the fractal dimensionality of the percolative backbone and $\nu$ the correlation length exponent. The derivation was made under the explicit assumption that the system is microscopically brittle, i.e. linearity between the stress and the strain of a bond up to its fracture. Inserting the known values of $T=3.96$ (see Ref. [8]) $d=2, d_{\mathrm{B}}=1.62$ (Ref. [7]) and $\nu=4 / 3$ one finds $T_{\mathrm{f}} \geqslant 2.12$, which is in agreement with our results. However, caution must be taken, if one recalls that our samples are at least not macroscopically brittle (Fig. 4).

Under similar assumptions, Bergman [2] has obtained both an upper and a lower bound on $T_{\mathrm{f}}$, namely :

$$
T-\nu z<T_{\mathrm{f}}<T-1
$$

where $z$ is the exponent that characterizes the shortest path $\xi_{c}$ between neighbouring nodes of the backbone (also known as the chemical path) $\xi_{\mathrm{c}} \sim$ $\xi^{z}$. Taking $z \simeq 1.10$ (see Ref. [9]) we find

$$
2.49<T_{\mathrm{f}}<2.96
$$

which is in good agreement with our results.

We now argue that this inequality is applicable not only to brittle materials as envisioned originally, but to a much wider class. The basic idea used to derive equation (2) was that in a diluted elastic network with both bond-stretching and bond-bending force constants, it is only the latter that are important near the percolation threshold $p_{\mathrm{c}}$. This idea, due to Kantor and Webman [10], explains not only the linear elastic properties near $p_{\mathrm{c}}$, but the fracture properties as well. In reference [2], a bond angle was assumed to rupture as soon as its angular distortion $\delta \phi$ exceeded some critical value $\delta \phi_{c}$, with the elastic properties remaining linear up to that point (this is the assumption of microscopic brittleness). Instead of that, we shall assume here that when $\delta \phi$ exceeds $\delta \phi_{\mathrm{c}}$ the bond angle does not rupture, but that its bending force constants drops discontinuously and irreversibly from its original value $m_{0}$ to a much smaller value $m_{1}$. In this way the inequality of equation (2) now refers to the plastic yield stress, exponent $T_{\mathrm{y}}$ i.e.

$$
\sigma_{\mathrm{y}} \sim\left(p_{\mathrm{c}}-p\right)^{T_{\mathrm{y}}}
$$

where $\sigma_{\mathrm{y}}$ is the value of macroscopic stress at which the material first undergoes irreversible plastic distortions. As the macroscopic stress applied to the system increases, a critical value is reached $\sigma_{\mathrm{y}}$ at which for the first time a bond angle undergoes this irreversible transition - this value is called the plastic yield stress. In order to estimate $\sigma_{y}$ for a diluted elastic network near $p_{\mathrm{c}}$, we invoke the linksnodes-blobs (LNB) picture for the percolating backbone. In that picture, the macroscopic intensive properties are determined by the properties of a typical link which connects two neighbouring nodes of the backbone, at a typical separation given by the correlation length $\xi \sim\left(p_{\mathrm{c}}-p\right)^{-\nu}$. When the ends of such a link are stretched (or compressed), most of the strain is carried by the singly connected bond angles (SCBA), and they will therefore be the first to exhibit nonlinear behaviour or undergo the irreversible plastic change described above. If only the SCBA's were distorted, then the angular strain of a SCBA would be related to the macroscopic strain $\varepsilon$ and to the end-to-end displacement of a link $\delta R$ by $L_{\ell} \delta \phi_{\mathrm{SCBA}} \sim \delta R / \xi \sim \varepsilon$, where $L_{\ell} \sim$ $\left(p_{\mathrm{c}}-p\right)^{-1}$ is the total number of SCBA's in a link. Since, in fact, the other bond angles and bond lengths may also be somewhat distorted, we get an inequality

$$
L_{\ell} \delta \phi_{\mathrm{SCBA}} \leqslant \delta R / \xi \sim \varepsilon .
$$

An inequality in the opposite sense is obtained if we replace $L_{\ell}$ in this equation by the length of a shortest path from end to end of a link $\xi^{z}$, namely

$$
\xi^{z} \delta \phi_{\mathrm{SCBA}} \geqslant \frac{\delta R}{\xi} \sim \varepsilon .
$$

Plastic yield will occur when $\delta \phi_{\mathrm{SCBA}}$ equals $\delta \phi_{\mathrm{c}}$, so that

$$
\begin{gathered}
\delta \phi_{\mathrm{c}}\left(p_{\mathrm{c}}-p\right)^{T-1} \leqslant \sigma_{\mathrm{y}} \sim \varepsilon\left(p_{\mathrm{c}}-p\right)^{T} \leqslant \\
\quad \leqslant \delta \phi_{\mathrm{c}}\left(p_{\mathrm{c}}-p\right)^{T-\nu z} \\
T-\nu z<T_{\mathrm{y}}<T-1 .
\end{gathered}
$$

We have thus regained the inequality of equation (2), but with $T_{\mathrm{f}}$ replaced by $T_{\mathrm{y}}$.

After one SCBA of a link has undergone the transition $m_{0} \rightarrow m_{1}$, it will carry a smaller stress, and consequently the stresses carried by other links will increase. Thus they too will reach the same kind of plastic threshold where one SCBA of each link undergoes the transition $m_{0} \rightarrow m_{1}$. If the macroscopic strain is increased slowly with time, a series of such discontinuous transitions should occur. At each transition the macroscopic stress jumps to a lower value and then increases continuously until the next transition. If the microscopic bending constants 
$m_{0}$ and critical angular strains $\delta \phi_{\mathrm{c}}$ have the same values for all bond angles, then the critical value of the macroscopic stress is expected to decrease monotonically for consecutive transitions. Thus, if an experiment is performed in which the stress is under control and is increased monotonically, then as soon as $\sigma_{\mathrm{y}}$ is reached, not only will plastic yield occur, but total fracture as well, i.e., $\sigma_{\mathrm{y}}=\sigma_{\mathrm{f}}$. If the values of $m_{0}$ and $\delta \phi_{\mathrm{c}}$ have a statistical distribution, we can expect deviations from the strict monotonicity rule. That is how we interpret the behaviour exhibited in figure 1 , where the first transition indeed occurs at the maximum stress, but the subsequent critical stress values are not entirely monotonic with time (and hence with strain). It is also conceivable that for a sufficiently broad distribution of the bond angle parameters $m_{0}, \delta \phi_{\mathrm{c}}$ the first plastic transition does not occur at the maximum value of stress. In that case $\sigma_{\mathrm{y}}<\sigma_{\mathrm{f}}$ and moreover the critical behaviour of the fracture process might then belong to a different universality class. Since in reference [3] it is reported that « the load at which fracture of the first bond occurs does not in general coincide with the maximum load in the load - displacement curve ", we would like to suggest that their system indeed had such a broad distribution of microscopic parameters. Consequently, the fact that they found a value of $T_{\mathrm{f}}=1.7 \pm 0.1$, which differs considerably from our value and is even outside the bounds of equation (3), may be due to the fact that their system belongs to a different universality class.

After an entire cross section of links of the LNB superlattice has undergone the plastic transition, the system can be stretched further until the $m_{1}$ bond angles become flat. If the system is stretched even beyond that point it can be expected to rupture into two separate and unconnected pieces. When this occurs, if only the $m_{1}$ bond angles are distorted then the absolute elongation of the system $L_{\mathrm{f}}$ should be of the order of $\xi$. Since in fact other angles and also bond lengths may be distorted, is only a lower bound on $L_{\mathrm{f}}$. If we define the critical strain exponent $T_{\mathrm{u}}$, as above by

$$
\varepsilon_{\mathrm{f}}=\frac{L_{\mathrm{f}}}{L} \sim\left(p_{\mathrm{c}}-p\right)^{-T_{\mathrm{u}}}
$$

where $L$ is the total length in the direction of stretching, then what we have found can be summarized as $L_{\mathrm{f}} \geqslant \xi, T_{\mathrm{u}} \geqslant \nu$. Since $\nu=4 / 3$ for $d=2$, this is in good agreement with our measurements (see Figs. 3 and 6).

We note that despite the fact that for total rupture to occur some links must achieve a relative strain on the order of 1 or more, it nevertheless follows from our picture that the macroscopic relative strain can be much less than 1 if the length $L$ of the system is much greater than $\xi$. This too is at least in qualitative agreement with our measurements (see Fig. 6).

In a recent article [11], it has been suggested that electrical breakdown thresholds, which are somewhat similar to the mechanical fracture threshold discussed here, vanish in the thermodynamic limit of an infinitely large system. We were unable to observe such an effect since we did not vary systematically the size of our samples.

The research at Tel-Aviv University (DJB) was supported in part by a grant from the Israel Academy of Sciences and Humanities and by a grant from the Soreq Nuclear Research Center of the Israel Atomic Energy Commission.

\section{References}

[1] a) Ray, P. and Chakrabarti, B. K., J. Phys. C 18 (1985) L 185, b) Preprint.

[2] Bergman, D. J., in Fragmentation, Form and Flow in Fractured Media, Ann. Israel Phys. Soc., R. Englman and Z. Jaeger Eds (1986) p. 266.

[3] Sieradzki, K. and Li Rong, Phys. Rev. Lett. 56 (1986) 2509.

[4] Benguigui, L., as in reference [2] p. 288.

[5] We note that $p_{\mathrm{c}}$ is reached when there is an infinite cluster of holes. Since the holes are punched on the sites of square lattice, we have $p_{\mathrm{c}} \simeq 0.596$ for site percolation on a square lattice.
[6] Benguigui, L., Phys. Rev. Lett. 53 (1984) 2028.

[7] Herrmann, H. J. and Stanley, H. E., Phys. Rev. Lett. 53 (1984) 1121.

[8] Zabolitzky, J. G., Bergman, D. J. and StaufFer, D., J. Stat. Phys. 44 (1986) 211.

[9] Hong, D. C., Havlin, S., Herrmann, H. J. and Stanley, H. E. Phys. Rev. B 30 (1984) 4083.

[10] Kantor, Y. and Webman, I., Phys. Rev. Lett. 52 (1984) 1891.

[11] Duxbury, P. M., Beale, P. D., Leath, P. L., Phys. Rev. Lett. 57 (1986) 1052. 